BMJ

Open

Gastroenterology

\title{
An evaluation of entecavir treatment among nucleos(t)ide-naïve Moroccan patients with chronic hepatitis B
}

\author{
Amal Chakkor, ${ }^{1}$ Fedoua Rouibaa, ${ }^{1}$ Safiaa Elaboudi, ${ }^{2}$ Aziz Aourarh, ${ }^{1}$
}

To cite: Chakkor $A$,

Rouibaa F, Elaboudi S, et al. An evaluation of entecavir treatment among nucleos $(\mathrm{t})$ ide-naïve Moroccan patients with chronic hepatitis B. BMJ Open Gastro 2016;3: e000081. doi:10.1136/ bmjgast-2016-000081

Received 27 January 2016 Revised 16 March 2016 Accepted 21 March 2016
'Gastroenterology Unit "I", Mohamed V Military Hospital, Mohamed V University, Rabat, Morocco

${ }^{2}$ Medecine "C" Unit, Ibn Sina University Hospital, Mohamed V University, Rabat, Morocco

Correspondence to Dr Amal Chakkor; amalchakkor@gmail.com

\section{ABSTRACT}

Objective: To analyse the efficacy and safety of entecavir (ETV) treatment in nucleos(t)ide (NUC)-naïve Moroccan patients with chronic hepatitis B.

Methods: We retrospectively analysed 41 NUT-naïve Moroccan patients with chronic hepatitis B who received ETV $0,5 \mathrm{mg} /$ day monotherapy for at least 3 months, of whom 3 were HBV envelope antigen (HbeAg) positive and 38 were $\mathrm{HBeAg}$ negative. The primary end point was the proportion of patients achieving virological response. Secondary end points included biochemical response (alanine transaminase (ALT) normalisation), serological response (HbeAg and HBV surface antigen (HBsAg) loss or seroconversion) and safety.

Results: The median follow-up duration was 74 weeks (48-144 weeks) and mean age was 43.8 years. Of 41 patients, 6 were primary non-responders and 2 achieved partial virological response at week 48, whereas 35 achieved undetectable hepatitis B virus (HBV) DNA at month 12. Viral suppression was maintained in $97.6 \%$ of patients after 3 years of ETV treatment. One patient experienced a virological breakthrough at month 12 of treatment. ALT normalisation occurred in $100 \%$ of the patients after 1 year of treatment. Only three patients in our study were $\mathrm{HbeAg}$ positive, of whom one has experienced seroconversion at month 12 of treatment. However, HBsAg loss or seroconversion was not achieved during the period of the study. No serious adverse event was reported.

Conclusions: These preliminary results showed that ETV is a safe and potent inhibitor of HBV in NUC-naïve Moroccan patients, but we need to observe more patients for a longer period of time, in order to assess the long-term effectiveness, safety, resistance profile and predictive factors for virological and serological response of ETV.

\section{BACKGROUND}

Chronic hepatitis B (CHB) is a major global health problem with more than 400 million people chronically infected in the world. ${ }^{1}$ Owing to its $25-40 \%$ associated lifetime risk of development of cirrhosis-related complications and hepatocellular carcinoma (HCC), ${ }^{2}$ it causes more than 600000 deaths every

\section{Summary box}

What is already known about this subject?

- Chronic hepatitis B (CHB) is an important disease problem in developing countries such as Morocco where HBV envelope antigennegative CHB is the most predominant type.

- Entecavir (ETV) is a potent inhibitor of hepatitis $B$ virus (HBV) with high barrier to resistance.

What are the new findings?

- Viral suppression was maintained in $97.6 \%$ of nucleos(t)ide-naïve Moroccan patients after 3 years of ETV treatment.

- All the patients had alanine transaminase normalisation after 1 year of treatment.

- Seroconversion occurred in $2.4 \%$ of the patients at month 12 of treatment.

How might it impact on clinical practice in the foreseeable future?

- ETV treatment is a highly effective anti-HBV treatment for Moroccan patients, with a favourable tolerability profile.

year $^{3}$ and currently represents $5-10 \%$ of liver transplantation indications. ${ }^{4}$ Morocco is considered a low endemic area thanks to the success of the vaccination programme; nevertheless, CHB still remains an important national disease burden. ${ }^{5}$

Antiviral therapy is an effective way of improving quality of life and survival by preventing disease progression and even reversing liver fibrosis. The end point of treatment is to ensure a degree of virological suppression that will then lead to biochemical remission, histological improvement and prevention of complications. ${ }^{6}$ CHB management has witnessed a paradigm shift in the past decade since the introduction of oral nucleotide/nucleoside analogues. Entecavir (ETV) is currently recommended as a first-line choice in current hepatitis B virus (HBV) treatment guidelines because of its potent viral suppression and large genetic barrier to resistance. $^{7}$ 
To the best of our knowledge, there are no previous published studies or data regarding ETV treatment in Morocco. In this study, we assessed the efficacy and safety of ETV on patients with CHB receiving ETV treatment in our unit.

\section{METHODS}

\section{Study population}

This retrospective longitudinal study collected consecutive patients from our outpatient clinic between January 2011 and December 2015. Patients were eligible if they were aged 18-70 years and had CHB defined by hepatitis B surface antigens (AGHBs) carriage beyond a 6-month period. As recommended by European Association for the study of the liver (EASL) practice guidelines, treatment indications were based mainly on the combination of three criteria: Serum HBV DNA levels $\geq 2000 \mathrm{IU} / \mathrm{mL}$, serum ALT levels above the upper limit of normal and severity of liver disease assessed by liver biopsy showing moderate to severe active nercoinflammation and/or at least moderate fibrosis. ${ }^{8}$

Patients were excluded from the study if they had hepatitis D virus, hepatitis C virus and HIV, hepatocellular carcinoma or comorbidities including alcoholic, autoimmune, metabolic liver disease with steatosis or steatohepatitis.

Among 65 patients treated with ETV daily for at least 3 months, 24 were excluded because of co-infection with Hepatitis $\mathrm{C}$ virus $(\mathrm{n}=11)$ and unavailable results for baseline and/or month 3 serums HBV DNA $(n=13)$. A total of 41 patients were included in this analysis and took ETV monotherapy $0.5 \mathrm{mg} /$ day (Bristol-Myers Squibb, Shanghai) for at least 3 months.

\section{Study design}

Pretherapeutic biological evaluation was performed systematically for each patient including: Routine haematological analysis, hepatobiliary enzymes, serological analysis, hepatic synthetic function, blood urea nitrogen, creatinine, thyroid-stimulating hormone ultrasensitive (TSHus) and blood glucose level. Serum ALT level and HBV DNA were assayed at baseline and every 36 months thereafter. HBV DNA follow-up used real-time PCR quantification assays. The same assay was used in the same patient to evaluate antiviral efficacy. The diagnosis of cirrhosis was based on histology. All the patients were carefully examined at each follow-up visit and asked to report any incidence of adverse events and to check treatment compliance.

\section{End points and definitions}

The primary end point was the proportion of patients achieving virological response defined as undetectable HBV DNA $(<100 \mathrm{IU} / \mathrm{mL})$ by a sensitive PCR assay evaluated every 3-6 months during therapy. Primary non-response is defined as less than $1 \log 10 \mathrm{IU} / \mathrm{mL}$ decrease in HBV DNA level from baseline at 3 months of therapy. Partial virological response is defined as a decrease in HBV DNA of more than $1 \log 10 \mathrm{IU} / \mathrm{mL}$ but detectable HBV DNA after at least 6 months of therapy in compliant patients. Virological breakthrough is defined as a confirmed increase in HBV DNA level of more than $1 \log 10 \mathrm{IU} / \mathrm{mL}$ compared to the nadir (lowest value) HBV DNA level on therapy. ${ }^{7}$ Secondary end points included biochemical response (ALT normalisation), serological response (HBV envelope antigen (HbeAg) and HBV surface antigen ( $\mathrm{HbsAg}$ ) loss or seroconversion) and treatment-related adverse events.

\section{Statistical analysis}

Data were analysed using the SPSS software V.20 (SPSS Inc, IBM). Quantitative data were presented as the mean $\pm \mathrm{SD}$. Categorical data were presented as counts and percentages. HBV DNA levels were presented as log transformation.

\section{RESULTS}

\section{Baseline characteristics of study patients}

The mean age of the 41 patients included in the study was $43.8 \pm 10.9$ years. Only $26 / 41$ of the patients $(63.4 \%)$ with CHB were diagnosed through a screening test (table 1$)$. There were 31 men $(75,6 \%)$ and 5 patients (12.2\%) with cirrhosis. The median baseline serum ALT level was 34 (26-255) IU/L and the serum HBV DNA level was $3.47(2.3-5.1) \log _{10} \mathrm{IU} / \mathrm{mL}$. The median treatment duration was 74 weeks (range, 48 to 144 weeks). Of 41 patients, $3(7.3 \%)$ were HBeAg-positive and 38 (92.7\%) were HBeAg-negative.

Table 1 Characteristics of patients at baseline

\begin{tabular}{llll}
\hline Baseline demographics & HBeAg-positive & HBeAg-negative & Total \\
\hline $\mathrm{n}(\%)$ & $3(7.3)$ & $38(92.7)$ & $41(100)$ \\
Male (\%) & $3(100)$ & $28(73.7)$ & $31(75.6)$ \\
Age (years, mean) & $41.7 \pm 14.4$ & $44 \pm 10.8$ & $43.8 \pm 10.9$ \\
Presence of cirrhosis (\%) & $1(33)$ & $4(10.5)$ & $5(12.2)$ \\
Interferon experienced ( $\mathrm{n}, \%)$ & $3(100)$ & $24(58.5)$ & $27(65.8)$ \\
Naïve patients ( $\mathrm{n}, \%)$ & $0(0)$ & $14(36.8)$ & $14(34.1)$ \\
ALT (U/L, median) & $40(30-42)$ & $31(26-255)$ & $34(26-255)$ \\
HBV DNA (log10 IU/mL) & $5(2.3-5.1)$ & $3(2.5-4.7)$ & $3.47(2.3-5.1)$ \\
ALT,valanine transaminase; HbeAg, HBV envelope antigen; HBV, hepatitis B virus. & &
\end{tabular}




\section{TREATMENT OUTCOMES}

\section{Virological response}

Six patients $(14.6 \%)$ were primary non-responders and 2 achieved partial virological response $(4.8 \%)$ at week 48 , whereas $35(85.3 \%)$ achieved undetectable HBV DNA at month 12. Viral suppression was maintained in $40 / 41$ patients $(97.6 \%)$ after 3 years of ETV treatment (figure 1). One patient (2.4\%) experienced a virological breakthrough at month 12 of treatment.

\section{Biochemical and serological response}

ALT normalisation occurred in $100 \%$ of the patients after 1 year of treatment (figure 1 ). Only $3 / 41$ patients in our study were HbeAg positive, of whom one experienced seroconversion at month 12 of treatment. However, HBsAg loss or seroconversion was not achieved during the period of the study.

\section{Safety and side effects}

During this study, there were no significant clinical side effects reported, especially no induced renal impairment and no lactic acidosis. Patients with liver cirrhosis did not develop any clinical decompensation or HCC during ETV therapy.

\section{DISCUSSION}

The main end point of chronic HBV treatment with nucleos(t)ide (NUC) is complete viral suppression. Once HBV DNA is cleared, continuing treatment may achieve HBeAg clearance and antiHBe seroconversion in $\mathrm{HBeAg}$-positive patients and less frequently $\mathrm{HbsAg}$ clearance and antiHBs seroconversion in $\mathrm{HBeAg}$-positive and negative patients. ${ }^{9}$ In the present study, the results of the 3-year analysis of 41 NUC-naive Moroccan patients with $\mathrm{CHB}$ were presented. Precore mutant $\mathrm{CHB}$ was found in $38(92.7 \%)$ of 41 patients. This finding agrees well with data reported in studies, confirming that it is the predominant type of $\mathrm{CHB}$ in the Mediterranean basin ${ }^{10}$ and thus in Morocco. ${ }^{11} \mathrm{HBeAg}$-negative CHB is a potentially severe disease that rapidly progresses to cirrhosis and related complications. ${ }^{12}$ Therefore, appropriate use of effective therapy is an important issue in the management of this group of patients.

There was obvious serum HBV DNA reduction and increased virological response at month 3 and month 6 , which confirm ETV to be a highly potent antiviral agent. The rate of ALT normalisation was increased over time too, which is consistent with previous results. The virological response was found to be $85,3 \%$ at the end of 12 months and more than $95 \%$ patients after 36 months of ETV treatment. In Spain, treatment for 12 months was associated with $82 \%$ HBV DNA negativisation, $26 \%$ HBeAg and 2\% HBsAg clearance rates in 190 NUC-naive patients. ${ }^{13}$ In Italy, treatment of 100 consecutive NUC-naïve patients reported 94\% HBV DNA negativisation, 33\% $\mathrm{HBeAg}$ and $15 \% \mathrm{HBsAg}$ clearances rates after 36 months of continuous ETV treatment. ${ }^{14}$ Results from Hong Kong showed that 222 treatment NUC-naïve patients achieved 92\% HBV DNA negativisation, 44\% HbeAg and 0, 45\% HbsAg clearance rates after 36 months of ETV treatment. ${ }^{15}$ All these studies showed a very low incidence of ETV resistance and virological breakthrough: $<1 \%$. In our study, one patient $(2,4 \%)$ had experienced a virological breakthrough at month 12 of treatment and was treated with ETV plus Adéfovir ADV (Ténofovir unavailable in Morocco).

Those present results are consistent with other experiences $^{16-18}$ showing increasing virological and biochemical response rates with continuous treatment. Controversially, they showed lower HBeAg clearance and seroconversion rates which emphasise the importance of long-term treatment. In addition, our study confirms that ETV treatment is safe as reported in clinical trials. ${ }^{19}$ There were no serious side effects and no patient discontinued ETV due to intolerance.

\section{CONCLUSION}

In summary, we acknowledge that this study has its limitations. Even though patients were prospectively followed, it is a retrospective study with a small sample
Figure 1 Virological, biochemical and serological responses according to ETV month of treatment. ALT,alanine transaminase; ETV, entecavir; HbeAg, HBV envelope antigen; HBV, hepatitis B virus; HbsAg, HBV surface antigen.

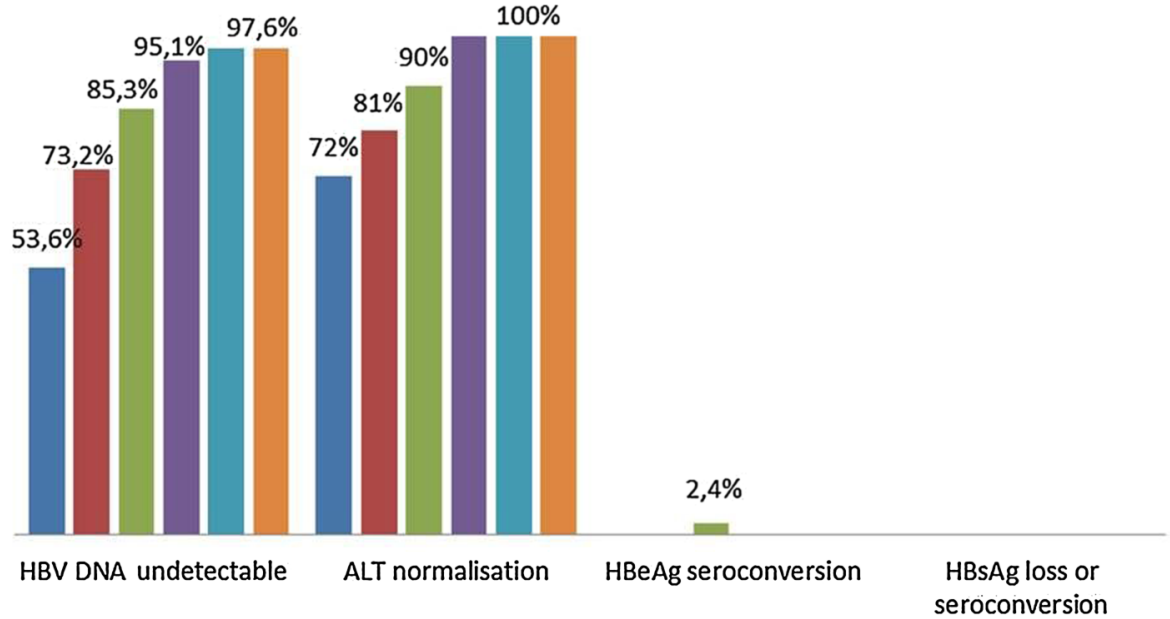

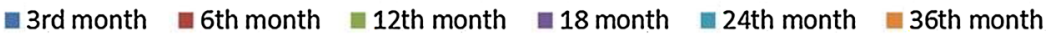


size. Also, we did not measure HbsAg levels at baseline and on-treatment response due to economic reasons (non-covered test by health insurance). In conclusion, we were able to demonstrate that ETV treatment is a highly effective anti-HBV treatment for Moroccan patients, with a favourable tolerability profile. This study has shown that ETV is a potent inhibitor of HBV, with greater efficacy in NUC-naïve Moroccan patients, but we need to observe more patients for a longer period, in order to assess the long-term effectiveness, safety, resistance profile and predictive factors for the virological and serological responses of ETV.

Competing interests None declared.

Ethics approval Faculty of Medicine and Pharmacy, Ethics Committee, Rabat, Morocco.

Provenance and peer review Not commissioned; externally peer reviewed.

Data sharing statement No additional data are available.

Open Access This is an Open Access article distributed in accordance with the Creative Commons Attribution Non Commercial (CC BY-NC 4.0) license, which permits others to distribute, remix, adapt, build upon this work noncommercially, and license their derivative works on different terms, provided the original work is properly cited and the use is non-commercial. See: http:// creativecommons.org/licenses/by-nc/4.0/

\section{REFERENCES}

1. McMahon BJ. Epidemiology and natural history of hepatitis B. Semin Liver Dis 2005;25(Suppl 1):3-8.

2. Yuen MF. Revisiting the natural history of chronic hepatitis B: impact of new concepts on clinical management. J Gastroenterol Hepatol 2007;22:973-6.

3. Parkin DM, Pisani P, Ferlay J. Estimates of the world wide incidence of 25 major cancers in 1990. Int J Cancer 1990;80:827-41.
4. Ganem D, Prince, AM. Hepatitis B virus infection-natural history and clinical consequences. N Engl J Med 2004;350:1118-29.

5. Sbaia A, Baha W, Ougabrai $\mathrm{H}$, et al. Prévalence de l'infection par le virus de l'hépatite $B$ et l'évaluation des facteurs de risque au Maroc. Pathol Biol 2012;60:e65-9.

6. European Association for the Study of the Liver. EASL clinical practice guidelines: management of chronic hepatitis B. J Hepatol 2009;50:227-42.

7. Osborn M. Safety and efficacy of entecavir for the treatment of chronic hepatitis B. Infect Drug Resist 2011;4:55-64.

8. European Association for the Study of the Liver. EASL clinical practice guidelines: management of chronic hepatitis $B$ virus infection. J Hepatol 2012;57:167-85.

9. Lok ASF, McMahon BJ. Chronic hepatitis B: update 2009. Hepatology 2009;50:661-2.

10. Rizzetto M. Response of pre-core mutant chronic hepatitis $B$ infection to lamivudine. J Med Virol 2000;61:398-402.

11. Kitab B, El Feydi AE, Afifi R, et al. Hepatitis B genotypes/ subgenotypes and MHR variants among Moroccan chronic carriers. $J$ Infect 2011;63:66-75.

12. Hadziyannis SJ, Vassilopoulos D. Hepatitis B e antigen-negative chronic hepatitis B. Hepatology 2013;46:17-624.

13. Buti M, Morillas RM, Prieto M, et al. Efficacy and safety of entecavir in clinical practice in treatment-naive Caucasian chronic hepatitis $B$ patients. Eur J Gastroenterol Hepatol 2012;24:535-42.

14. Marengo A, Bitetto D, D'Avolio A, et al. Clinical and virological response to entecavir in HBV-related chronic hepatitis or cirrhosis: data from the clinical practice in a single-centre cohort. Antivir Ther (Lond) 2013;18:87-94.

15. Yuen MF, Seto WK, Fung J, et al. Three years of continuous entecavir therapy in treatment-naïve chronic hepatitis B patients: VIRAL suppression, viral resistance, and clinical safety. Am J Gastroenterol 2011;106:1264-71.

16. Luo J, Li X, Wu Y, et al. Efficacy of entecavir treatment for up to 5 years in nucleos(t)ide-naïve chronic hepatitis $\mathrm{B}$ patients in real life. Int J Med Sci 2013;10:427-33.

17. Yao GB, Ren H, Xu DZ, et al. Results of 3 years of continuous entecavir treatment in nucleos(t)ide-naive chronic hepatitis B patients. Zhonghua Gan Zang Bing Za Zhi 2009;17:881-6.

18. Ono A, Suzuki F, Kawamura Y. Long-term continuous entecavir therapy in nucleos(t)ide-naïve chronic hepatitis B patients. J Hepatol 2012;57:508-14.

19. Kobashi H. Efficacy and safety of entecavir in nucleoside-naive, chronic hepatitis B patients: phase II clinical study in Japan. J Gastroenterol Hepatol 2009;24:255-61. 